\title{
Piezoresistive effect in epoxy-graphite composites
}

\author{
N. Serra, T. Maeder, P. Ryser \\ Laboratoire de Production Microtechnique, Ecole Polytechnique Fédérale de Lausanne, Station 17, CH-1015 Lausanne, Switzerland \\ http://1pm.epfl.ch; nathalie.serra@epfl.ch; thomas.maeder@epfl.ch \\ Original version: Procedia Engineering 25 (Proc. Eurosensors XXV, September 4-7, 2011, Athens, Greece), 235-238, 2011 \\ (C) 2011 Published by Elsevier Ltd ; http://hdl.handle.net/10.1016/j.proeng.2011.12.058
}

\begin{abstract}
In this work, we investigate the piezoresistive response of epoxy-graphite composites. A resistive thick-film Wheatstone bridge is deposited by screen-printing onto an alumina beam, a weight is then applied on the tip of the beam and the resulting electrical signal response is recorded, allowing the calculation of the gauge factor. The characterization was made at room temperature, $60^{\circ} \mathrm{C}$ and $100^{\circ} \mathrm{C}$ for different matrixes (epoxies with different glass transition temperatures, $\mathrm{Tg}$ ) and particles sizes $(4 \mu \mathrm{m}$ and $15 \mu \mathrm{m})$. The creep of the signal in time and temperature was also observed. The present work shows also the tremendous effect of $\mathrm{Tg}$ on piezoresistive behavior: the high $\mathrm{Tg}$ epoxy demonstrating better stability in time and temperature than the other one.
\end{abstract}

Keywords: piezoresistivity; epoxy-graphite composite; creep

\section{Nomenclature}

$T_{g}$

$G F, G F_{L}, G F_{T}$

glass transition temperature

gauge factor, longitudinal $\left(G F_{L}\right)$ and transverse $\left(G F_{T}\right)$

\section{Introduction}

Percolative nanomaterials find increasing use in industrial applications. For instance, thick-film resistors, which essentially consist of conductive oxide nanopowder in an insulating glassy matrix forming a paste and can be printed on substrate [2], are used in electronic circuits and may be applied to sensors for mechanical and chemical quantities and temperature. Although these materials are of great interest for integrating sensing functions in high-reliability technological devices, variants having lower processing temperatures compatible with organic substrates are also sought for a wider range of applications. From this perspective, the use of polymer/graphite composites featuring good processability and low-cost materials is very promising. Several properties of the material used, such as mechanical or electrical (depending on the type of sensor) must be therefore considered.

In a previous work [1], we studied the conductivity properties of epoxy-graphite composites, regarding particle size and $T_{g}$ effects, finally leading to the manufacture of micro-heaters based on these pastes. To continue the characterization of our pastes, we observed the piezoresistive behavior, which is of high interest for mechanical sensors. Piezoresistivity has already been studied for thick-films resistors [3-4]. However it has also been demonstrated that the effects depends strongly on the conductive properties of the filler [4]. As little work has been done concerning standard graphite, most of the literature dealing with graphite fibers or expanded graphite [5-6], it can be really interesting to investigate the piezoresistive response of thick-film epoxy-graphite composite. The characterization was completed by the study of the creep of our beams in temperature.

\section{Experimental}

Alumina test beams were produced using screen-printing process. Different resistive pastes are deposited on the substrate, forming a Wheatstone bridge. A weight is then applied on the tip of the beam and the resulting electrical signal response is recorded, leading, after calculation, to the gauge factor (longitudinal and transverse). The particle size effect was investigated. Two kinds of graphite were tested as filler: KS4 and KS15. Both variants have an ellipsoidal shape and come from Timcal, Switzerland, KS4 (resp. KS15) meaning that $95 \%$ of the particles have a large axis smaller than $4 \mu \mathrm{m}$ (resp. $15 \mu \mathrm{m}$ ). Two different epoxies were also tested as matrix for the composites; they were chosen with different $\mathrm{Tg}$ values, in order to see the impact in creep stability. Table 1 sums up the characteristics of these epoxies. Pastes were made in each case for different volumic concentration of filler: $4 \%, 6 \%, 10 \%, 12 \%, 15 \%$ and $20 \%$ in order to change the conductivity of the composite and observed the change in the piezoresistivity response. Measurements were made at room temperature, $65^{\circ} \mathrm{C}$ and $100^{\circ} \mathrm{C}$ for both matrixes. The gauge factor is defined as: 


$$
G F=(\Delta R / R) /(\Delta l / l)=(\Delta R / R) / \varepsilon
$$

where $(\Delta R / R)$ is the relative change in resistance and $\varepsilon$ the strain with $\varepsilon=\Delta l / l$

$\mathrm{GF}_{\mathrm{L}}$ is the case where the current flows along the length of the resistor (i.e. the current is parallel to the applied strain) and $\mathrm{GF}_{\mathrm{T}}$ where the current flows along the width of the resistor (i.e. current perpendicular to the applied strain). Both factors were determined in our experiments. However only the $\mathrm{GF}_{\mathrm{L}}$ are discussed here, the effect being the same for $\mathrm{GF}_{\mathrm{T}}$ but the error is higher due to geometric factor. Finally, the creep of the signal vs. time was observed at different temperatures using the same beams. Measurements were made at $25^{\circ} \mathrm{C}, 40^{\circ} \mathrm{C}, 50^{\circ} \mathrm{C}, 60^{\circ} \mathrm{C}, 70^{\circ} \mathrm{C}, 80^{\circ} \mathrm{C}$ and $90^{\circ} \mathrm{C}$ for one complete cycle (unloading/loading/unloading).

\section{Results}

\subsection{Piezoresistive behavior}

A first series of experiments was made to determine the particle size effect on piezoresistivity. Fig 1a reports the results of the $\mathrm{GF}_{\mathrm{L}}$ at room temperature vs. volumic concentration for KS4 and KS15, the matrix being the epoxy Epotek 377. They show that with the smaller particles, the gauge factor is somewhat higher. For a better understanding of the phenomenon, Fig 1.b presents the results of piezoresistivity vs. resistivity. A higher resistivity is linked to a higher gauge factor [3]. In the case of bigger particles, the GF is higher for a same resistivity. This can be explained by the tunneling effect: smaller particles will present more tunnel junctions, but will be closer, leading to a smaller GF. The results are not totally in agreement with theory, this may be explained by the morphology of particles which are not perfectly spherical and their closeness in size.

A second series of experiments was performed in order to see the impact of matrix thermomechanical stability on the samples. The gauge factor was calculated at room temperature, $65^{\circ} \mathrm{C}$ and $100^{\circ} \mathrm{C}$. The corresponding results are reported in Fig 2. When the temperature is increased and reaches the area of $T_{g}$, several effects are in competition: dilatation of the epoxy in the $\mathrm{z}$ direction with the graphite in compression, and beam in tension. This leads to high strains inside the material, thus instability of the gauge factor. With the high $\mathrm{T}_{\mathrm{g}}$ epoxy, strains and matrix relaxation effects are lower leading to an improved stability.

\subsection{Study of the piezoresistivity creep}

Using the same beam, the creep of the signal was observed in time. Fig 3 presents the curves obtained at $12 \%$ vol. graphite $\mathrm{KS} 15$ for the two epoxies at $25^{\circ} \mathrm{C}, 50^{\circ} \mathrm{C}$ and $80^{\circ} \mathrm{C}$. When the temperature is raised close to the $\mathrm{T}_{\mathrm{g}}$ of the Epotek 377, the material is disturbed due to higher dilatation of the matrix and relaxation effects, leading to strong instabilities (drifts) of the signal. On the other hand, we can observe that the Martens Plus, while not perfect, expectedly exhibits a better stability regarding the creep.

\section{Conclusion}

The piezoresistivity response of thick-film epoxy-graphite composite was studied. We demonstrate that particle size has a more important effect than in resistivity measurements, due to tunneling effect. We also once more highlighted the role of $T_{g}$ on mechanical stability, which can be a problem, as the material will dilate and rapidly relax around this temperature. Finally, the creep was observed at different temperatures. We can see that even at room temperature, the higher $T_{g}$ epoxy, though not perfect, presents better stability in time. These results are therefore promising for application to low-cost mechanical sensors.

\section{Acknowledgements}

The authors would like to thank Timcal for the graphite powders, and Mathias Garcin for manufacturing the samples. This work was partly financed by the Swiss CTI promotion agency.

\section{References}

[1] N. Serra, T. Maeder, P. Lemaire, P. Ryser. Formulation of composite resistive pastes for fabrication of microheaters. Sensors and Actuators A: Physical 2010;162:367-372

[2] N.M. White, J.D. Tuner. Thick-film sensors: past, present and future: Measurement Science and Technology 1997;8:1-20.

[3] S. Vionnet Menot. Low firing temperature thick-film piezoresistive composites-Properties and conduction mechanism. Thesis $\mathrm{n}^{\circ} 3290,2005$, Ecole Polytechnique Fédérale de Lausanne

[4] C. Canali, D. Malavasi, B. Morten, M. Prudenziati, A. Taroni. Piezoresistive effects in thick-film resistors. Journal of Applied Physics 1980;51:3282-3288

[5] F. Carmona, R. Canet, P. Delhaes. Piezoresistivity of heterogeneous solids. Journal of Applied Physics 1987;61:2550-2557

[6] S. Qu, S-C Wong. Piezoresistive behavior of polymer reinforced by expanded graphite. Composites Science and Technology 2007;67:231-237 
Table 1. Main properties of epoxy resins (supplier data)

\begin{tabular}{llll}
\hline Name & Supplier & $T_{g}\left({ }^{\circ} \mathrm{C}\right)$ & Curing Schedule \\
\hline Epotek 377 & PolyScience AG & $\approx 90$ & $2 \mathrm{~h} @ 150^{\circ} \mathrm{C}$ \\
Martens Plus & Swiss Composite & $\approx 200$ & $24 \mathrm{~h} @ 100^{\circ} \mathrm{C}+15 \mathrm{~h} @ 230^{\circ} \mathrm{C}$ \\
\hline
\end{tabular}

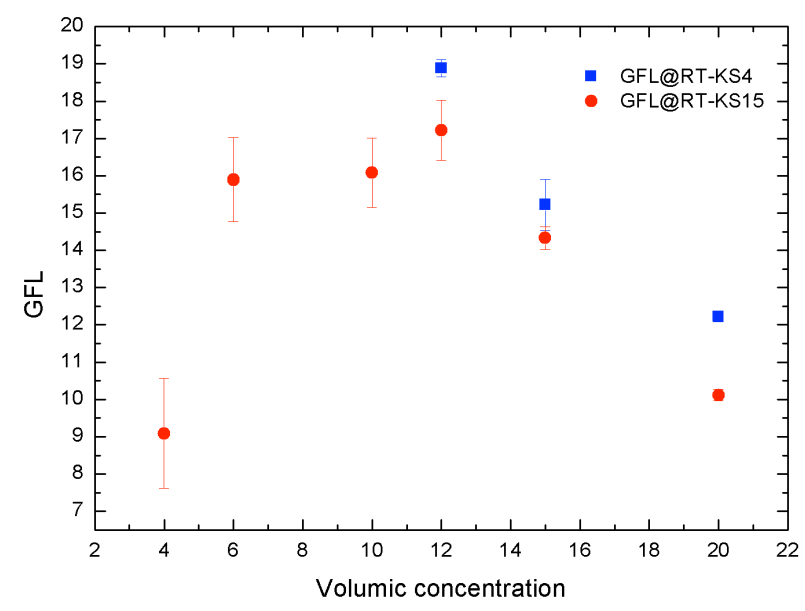

(a)

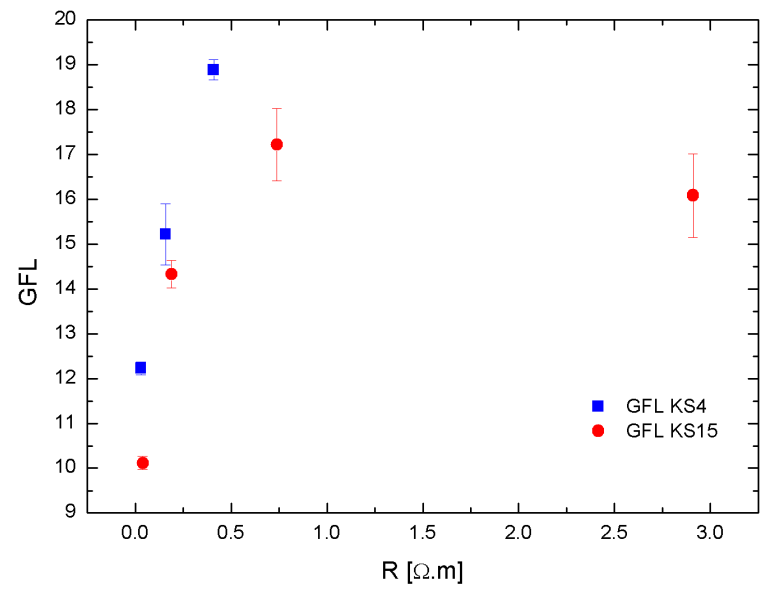

(b)

Fig 1. Effect of particle size on the GFL vs. graphite volumic concentration (a) and vs. resistivity (b) (matrix: Epotek 377, particles: KS4 and KS15)

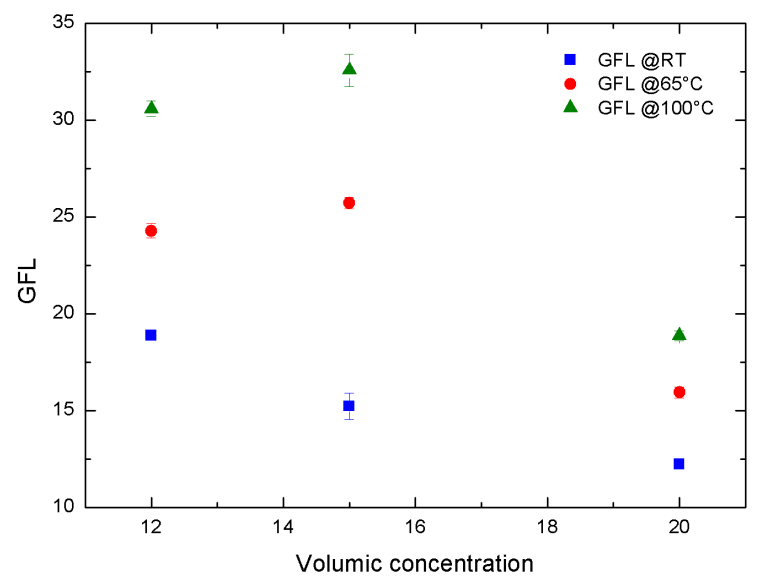

(a)

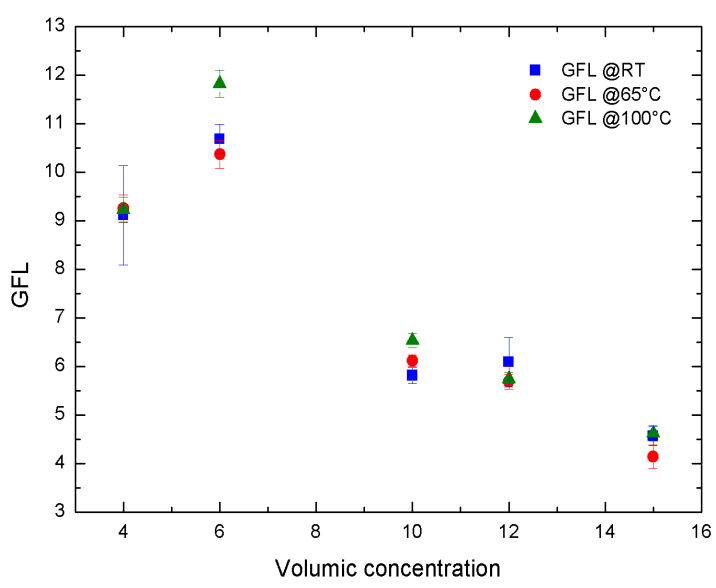

(b)

Fig 2. Longitidunal gauge factor at $\mathrm{RT}, 65^{\circ} \mathrm{C}$ and $100^{\circ} \mathrm{C}$ vs. graphite volumic concentration for Epotek 377 (a) and Martens Plus (b) 


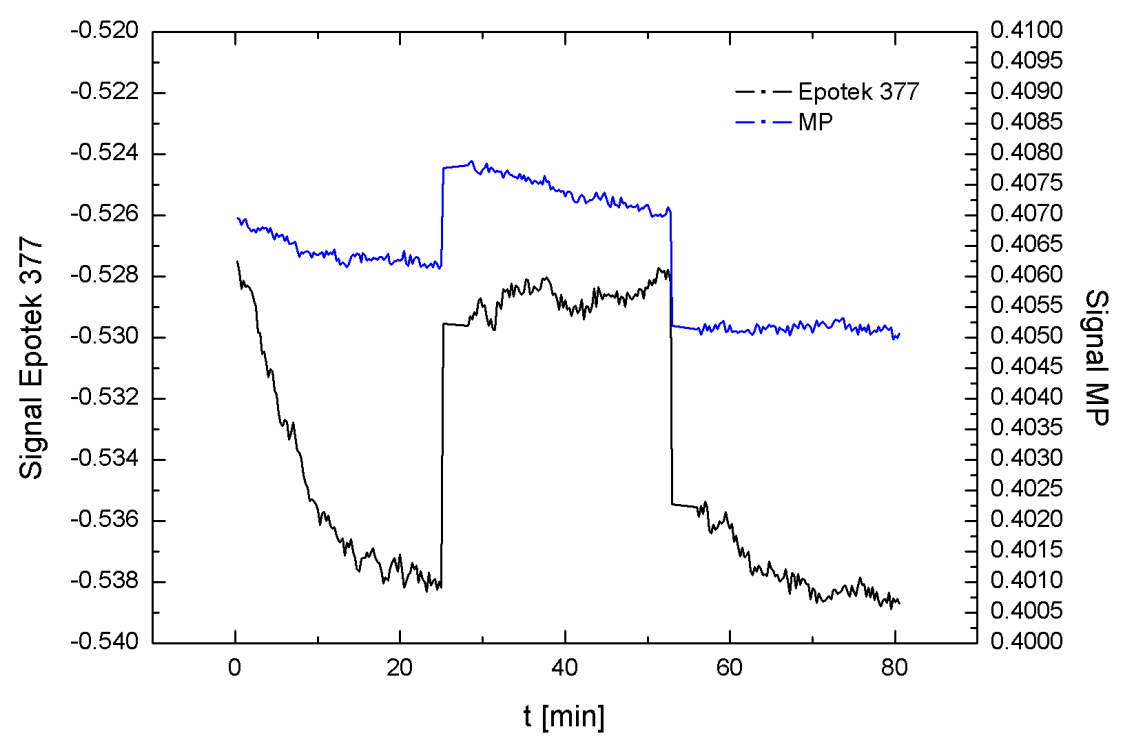

(a)

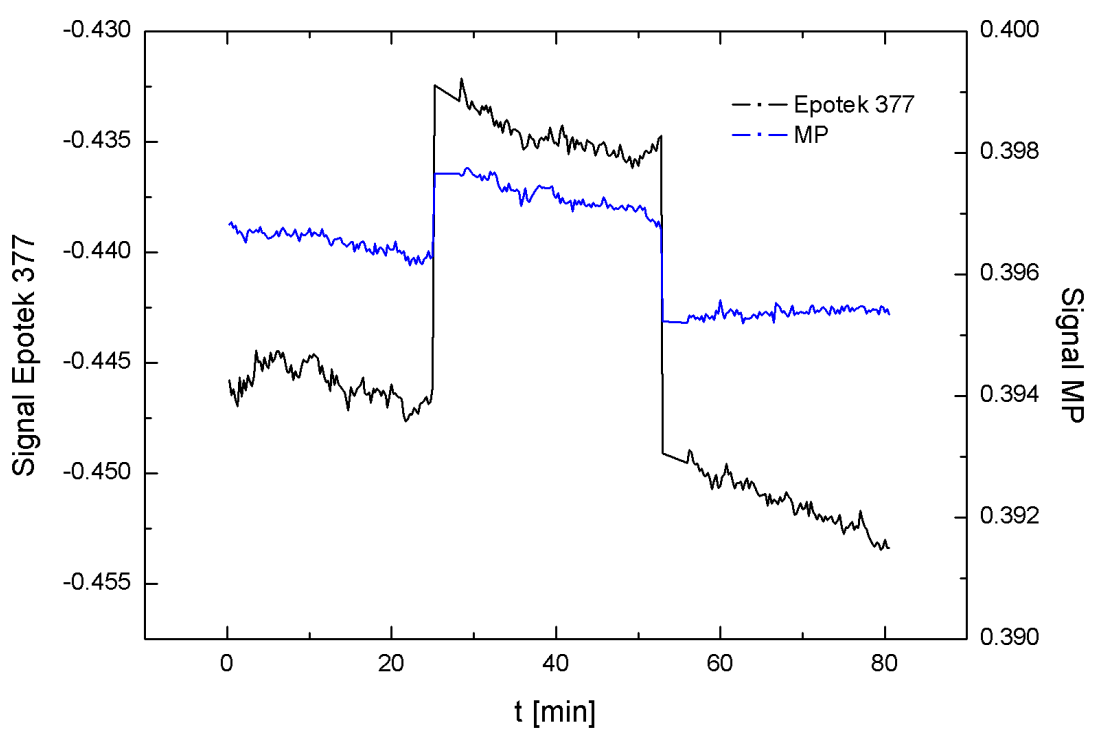

(b)

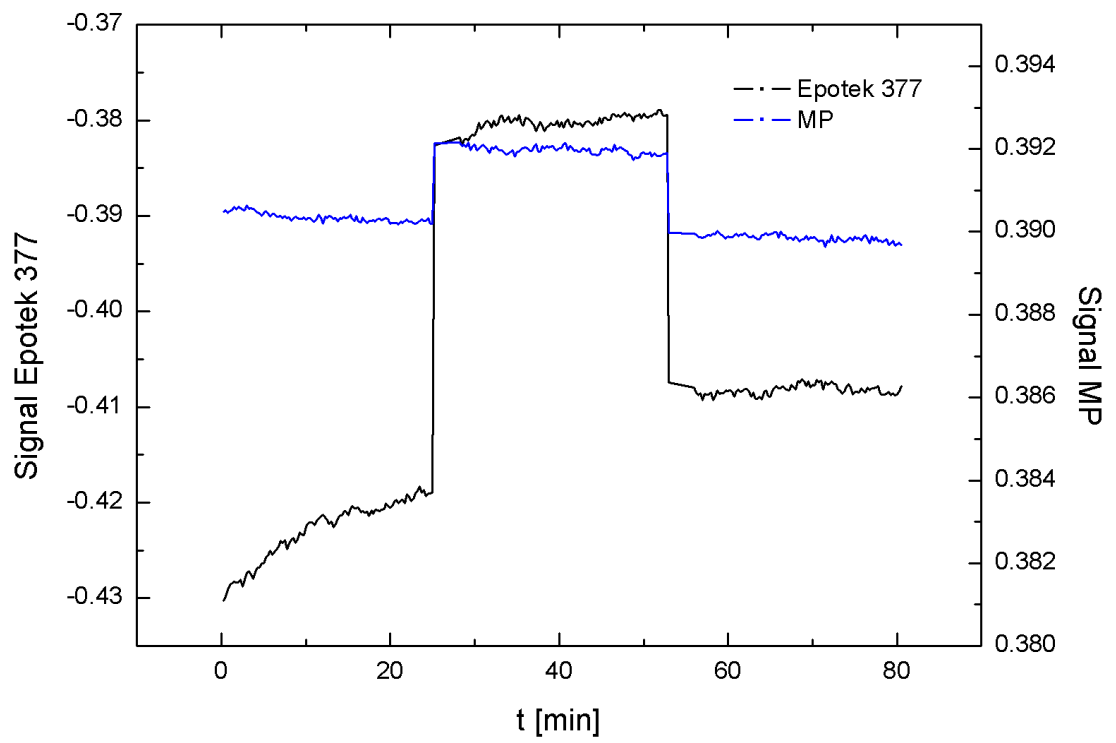

(c)

Fig 3. Signal vs. time for $12 \%$ vol of graphite in Epotek 377 and Martens Plus at $25^{\circ} \mathrm{C} \mathrm{(a),} 50^{\circ} \mathrm{C}(\mathrm{b})$ and $80^{\circ} \mathrm{C}(\mathrm{c})$ 